\title{
液态金属催化剂：二维材料的点金石
}

\author{
曾梦琪张 涛 谭丽芳 付 䂞 ${ }^{*}$ \\ (武汉大学化学与分子科学学院, 武汉 430072)
}

\begin{abstract}
摘要: 由于石墨烯等二维材料具有独特的结构与优异的性能, 其在众多新型电子器件的构建中具有重要的应 用前景。然而, 其可控生长仍然存在诸多挑战性的问题, 这也是制约这类明星材料真正迈向应用的瓶颈所 在。化学气相沉积法(CVD) 是目前可控制备高质量石墨烯最有效的方法, 其中催化基底的设计尤为重要, 这 将直接决定 CVD 最为核心的两个过程: 催化和传质。相较于改变催化剂的化学组成, 近年来我们发现改变 催化剂的物态一由固态到液态, 对石墨烯等二维材料的 CVD 过程有质的改变和提升。与固态基底相比, 液态基底具有更松散的原子排列、更剧烈的原子迁移, 使得液面平滑而各向同性, 液相可流动且可包埋异质 原子。这使得液态金属在催化石墨烯等二维材料及其异质结生长时表现出很多独特的行为, 比如层数严格自 限制、超快的生长速度、晶粒拼接平滑等。更重要的是, 基底的液态特性给二维材料的自组装和转移带来了 突破, 实乃二维材料的点金石。本文将梳理液态金属催化剂上二维材料的生长、组装与转移行为, 这些关键 技术的突破将为二维材料迈向真正应用奠定坚实的基础。
\end{abstract}

关键词: 液态金属; 二维材料; 生长; 组装; 转移

中图分类号: 0647

\section{Liquid Metal Catalyst: Philosopher's Stone of Two-Dimensional Materials}

\author{
ZENG Meng-Qi ZHANG Tao TAN Li-Fang FU Lei* \\ (College of Chemistry and Molecular Science, Wuhan University, Wuhan 430072, P. R. China)
}

\begin{abstract}
Graphene and graphene-like two-dimensional (2D) materials exhibit broad prospects for application in emerging electronics owing to their unique structure and excellent properties. However, there are still many challenges facing the achievement of controllable growth, which is the main bottleneck that limits the practical application of these materials. Chemical vapor deposition (CVD) is the most effective method for the controllable growth of high-quality graphene, in which the design of the catalytic substrate catches the most attention because it directly determines the two most significant basal processes_—catalyzation and mass transfer. Recently, compared with the selection of the chemical composition of the catalyst, the change of the physical state of the catalyst from a solid phase to liquid phase is expected to lead to a qualitative change and improvement in the CVD of graphene and graphene-like two-dimensional materials. Unlike solid substrates, liquid substrates exhibit a loose atomic arrangement and intense atom movement, which contribute to a smooth and isotropic liquid surface and a fluidic liquid phase that can embed heteroatoms. Therefore, liquid metal shows many unique behaviors during the catalyzation of the growth of graphene, graphene-like two dimensional materials, and their heterostructures, such as strict self-limitation, ultra-fast growth, and smooth stitching of grains. More importantly, the rheological properties of a liquid substrate can even facilitate the self-assembly and transfer of 2D materials grown on it, in which the liquid metal substrate can be regarded as the 'philosopher's stone'. This feature article
\end{abstract}

Received: October 10, 2016; Revised: November 15, 2016; Published online: November 15, 2016.

*Corresponding author. Email: leifu@whu.edu.cn; Tel: +86-27-68755687.

The project was supported by the National Natural Science Foundation of China $(51322209,21473124,21673161)$.

国家自然科学基金(51322209, 21473124, 21673161)资助项目

(c) Editorial office of Acta Physico-Chimica Sinica 
summarizes the growth, assembly, and transfer behavior of 2D materials on liquid metal catalysts. These primary technology developments will establish a solid foundation for the practical application of 2D materials.

Key Words: Liquid metal; Two-dimensional material; Growth; Assembly; Transfer

\section{1 引言}

维度减少对电子造成的空间限域导致二维材 料表现出与块体材料截然不同的物理化学性质。 这样一个庞大的材料家族的成员众多, 包括金 属、半金属、绝缘体和带隙跨度宽(从紫外光区到 红外光区)的半导体 (含直接带隙与间接带隙), 表 现出非常广泛的电学性质 ${ }^{1}$ 。可以预见的是, 石墨 烯等二维材料将在未来的纳电子器件、光电器件 以及新型超薄和柔性器件中扮演重要的角色, 它 们有望在很多关键技术领域带来质的而非仅仅量 的突破。然而, 这类材料是否能充分施展天赋取 决于能否实现可控制备, 比如所得材料的层数均 匀性和晶畴大小将直接决定其实际性能。以石墨 烯为例, 单层时其带隙为零 ${ }^{2}$; 对双层石墨烯施加 一个电场, 其带隙将连续可调 ${ }^{3,4}$; 而层数增加至三 层时, 其将表现出半金属性质 ${ }^{3,5}$, 更多层数的石墨 烯(大于十层)则基本与块体石墨无异了 ${ }^{6}$ 。此外, 二维材料晶界处的缺陷会使得材料的电学性能、 力学性能呈指数下降, 只有结构完美的二维材料 才能最大程度地发挥其优异的本征性质 ${ }^{7}$ 。截止目 前, 制备二维材料的主流方法主要包括剥离法和
化学气相沉积法(CVD), 其中后者被认为是能同时 实现大面积和高质量的有效方法。尽管二维材料 的制备技术一直在发展, 但如何使得其各项性质 和性能在量产过程(如以能源为导向的特定应用所 要求的大尺寸制备以及晶圆级尺度的集成等)中保 持在较高水平，仍是一个巨大的挑战 8

在 CVD 过程中, 催化基底的化学组成和物理 状态往往发挥着非常重要的作用, 通过影响吸 附、分解、扩散、表面反应、成核和生长等基元 步骤, 它们决定了前驱体的热解效率、材料的沉 积速率和最终产物的质量等。传统制备二维材料 薄膜或单晶是在刚性的固态基底上进行的，固态 基底表面晶体缺陷多且分布随机、原子排列各向 异性、质点往往被束缚、扩散受限, 这导致二维 材料成核随机且密度高, 获得的材料晶畴小而层 数不均, 而且由于传质缓慢, 生长效率也很低。 为了解决这些问题, 研究者们投入了大量精力, 致力于催化组份的设计和对表面形貌进行优化, 试图调控基元过程, 获得高质量层数均匀的二维 材料。然而结果往往不甚理想, 且重现性不佳。 近年来, 我们提出采用液态金属催化剂生长二维

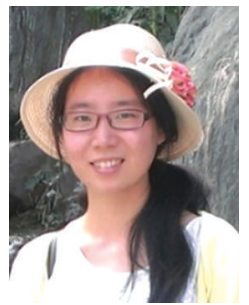

曾梦琪，1991年出生。2009-2013 年本科就读于武汉大学化学与分子 科学学院, 现为武汉大学博士研究 生。主要研究方向为液态金属催化 剂上石墨烯的可控生长与转移。

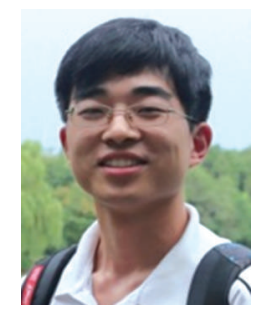

张涛，1991年出生。2010-2014年 本科就读于武汉大学化学与分子科 学学院, 现为武汉大学博士研究 生。主要研究方向为二维材料及其 异质结的可控制备。

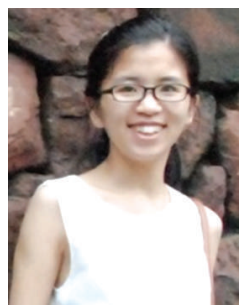

谭丽芳，1989年出生。2007-2011 年本科就读于湖北大学化学化工学 院, 现为武汉大学博士研究生。主 要研究方向为绝缘基底上石墨烯的 可控生长与应用。

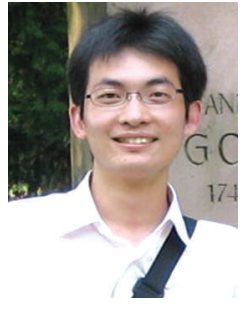

付磊, 2001 年于武汉大学化学与分 子科学学院获得学士学位, 2006年 于中国科学院化学研究所获得博士 学位。2006年加入美国洛斯阿拉莫 斯国家实验室开始独立从事研究工 作，2007年-2011 年任北京大学副 研究员, 2011 年至今任武汉大学化学与分子科学 学院教授、博士生导师。主要研究方向为二维材 料的可控生长及其在能源领域的应用。 
材料, 打破了这一已经僵化了很久的局面。物态 转变带来的传质和催化行为的变革给二维材料的 生长注入了新的活力。当金属处于熔融状态时, 原子的热运动剧烈, 热迁移效应显著, 使得表面 趋近各向同性; 体相原子空位多, 利于包埋异质 原子, 且易于切变; 流动性好, 导致传质迅速。 这使得二维材料在液态金属上生长时表现出独特 的层数自限制、生长速度快、晶粒拼接平滑等行 为。此外, 液态金属中自由电子的移动范围更 大, 在这样一个富含自由电子的表面, 化学吸附 分子的共价键更易形成, 这使其表现出更高的催 化活性, 在催化异质结生长时也表现出独到的优 势。更重要的是, 基底的液态特性给二维材料的 自组装和转移也带来了突破, 这将为二维材料迈 向真正应用奠定坚实的基础。

\section{2 二维材料生长的催化剂}

催化剂在二维材料的生长中起着至关重要的 作用。以石墨烯为例, 金属在与反应物接触时, 可以通过改变氧化态或者形成合适的中间产物以 提供低的反应能量通道等方式降低反应活化能, 实现催化作用。而根据催化剂的电子排布不同, 其参与反应的原子轨道可以是部分填充的 $d$ 轨道 (如镍 $(\mathrm{Ni})^{9,10}$ 、铁 $(\mathrm{Fe})^{11}$ 、铱 $(\mathrm{Ir})$ 等 $)^{12}$, 也可以是外层 $s$ 轨道(如铜 $\left.(\mathrm{Cu})^{13,14}\right)$ 和 $p$ 轨道 $\left(\right.$ 如镓 $\left.(\mathrm{Ga})^{15}\right)$ 。除了催化 活性以外, 金属与石墨烯的晶格失配度和碳溶解 度等也会影响其上石墨烯的生长。比如, 相较于 碳溶解度高的 $\mathrm{Ni}$, 碳溶解度极低的 $\mathrm{Cu}$ 更容易可控 地生长均匀层数的石墨烯 ${ }^{9,13}$ 。金属合金策略, 比 如 $\mathrm{Cu}-\mathrm{Ni}^{16,17} 、 \mathrm{Au}-\mathrm{Ni}^{18} 、 \mathrm{Ni}-\mathrm{Mo}^{19}$ 等, 也被广泛用于 调控石墨烯的 CVD 过程, 以实现高质量均匀层数 的石墨烯的可控制备。此外, 一些非金属, 比如 稳定的间隙金属碳化物也能催化生长石墨烯 ${ }^{20}$ 。

除了催化剂的化学组成外, 催化剂的表面结 构、形貌与物态同样值得关注 ${ }^{21,22}$ 。对于常用的固 态金属基底而言，虽然其内部的原子具有一定的 排列规律, 但其表面往往存在大量的缺陷, 比如 晶界。CVD生长石墨烯时, 碳原子通常会在晶界 处优先成核生长, 体相溶解的碳在降温时也优先 在晶界处析出, 因此多晶金属表面的平整度将影 响成核点的多少以及析出的均匀性, 从而决定石 墨烯的畴区大小以及层数均匀度。固态金属催化 基底的表面平整化成为提高二维材料质量的努力
方向。为此, 人们开始寻求各种方法对多晶金属 䇴进行表面处理, 以得到更为平整、更接近单晶 的表面, 包括热退火、电抛光、化学抛光、氧气 诱导重构等等 ${ }^{23-26}$ 。然而结果却不尽人意, 更有效 的表面平整化策略亟待提出。单晶金属基底 ${ }^{27,28}$ 由 于平整的表面以及无晶界优势, 一度成为研究热 点, 但昂贵的价格却宣告其难以获得大规模应 用。金属合金虽然在控制石墨烯层数和均匀性方 面具有优势 ${ }^{19}$, 但获得的石墨烯晶畴过小, 导致石 墨烯的迁移率等性能急剧下降。

仿佛 “山穷水尽” 时, 液态金属进入了我们 的视线。在熔融状态下, 金属原子排列的规律性 较弱, 整体表现出一种短程有序、长程无序的特 性, 从而能有效地减少表面的晶界等缺陷, 提供 一个各向同性的表面; 而且由于液态催化剂中原 子相对自由的热运动, 电子的移动范围也有所扩 大, 这使其具有与通常的固态催化剂不同的催化 行为。故而我们可以期待, 液态金属独特的催化 和传质行为将有助于实现二维材料生长的可控生 长。

\section{3 液态金属上二维材料的生长}

\section{1 液态金属上二维材料的层数控制}

对于二维材料的生长而言, 最基础、最重要 的便是对其层数的控制。下面将以石墨烯的生长 为例, 具体介绍液态金属催化剂在石墨烯层数控 制方面的优势。

石墨烯通常倾向于在金属表面的晶界等缺陷 处成核, 进而生长出厚层。当液态金属作为石墨 烯的生长基底时, 可以很大程度上解决石墨烯层 数不均匀的问题。这是因为, 液态金属表面并不 存在晶界, 而且非常均匀, 这有利于抑制石墨烯 的不均匀成核。本课题组首次利用液态金属镓 $(\mathrm{Ga})$ 获得了高质量均匀单层单晶石墨烯(图 1) ${ }^{15}$ 。图 2(ae) 展示了该方法制得的大面积高质量均匀石墨烯薄 膜。由于 $\mathrm{Ga}$ 表面具有较低的蒸气压, 这使得液态 $\mathrm{Ga}$ 表面极其均匀平滑, 这有利于降低石墨烯的成 核密度从而生长出高质量的石墨烯。液态 $\mathrm{Ga}$ 表面 石墨烯的成核密度低至 $1 / 1000 \mu \mathrm{m}^{-2}$, 为固态 $\mathrm{Ga}$ 上 的十分之一。结果表明, 在液态 $\mathrm{Ga}$ 表面生长的石 墨烯极其均匀, 单晶迁移率可达 $7400 \mathrm{~cm}^{2} \cdot \mathrm{V}^{-1} \cdot \mathrm{s}^{-1}$ (图 2(f))。

除了 $\mathrm{Ga}$ 以外, 我们还系统地研究了其他呈熔 


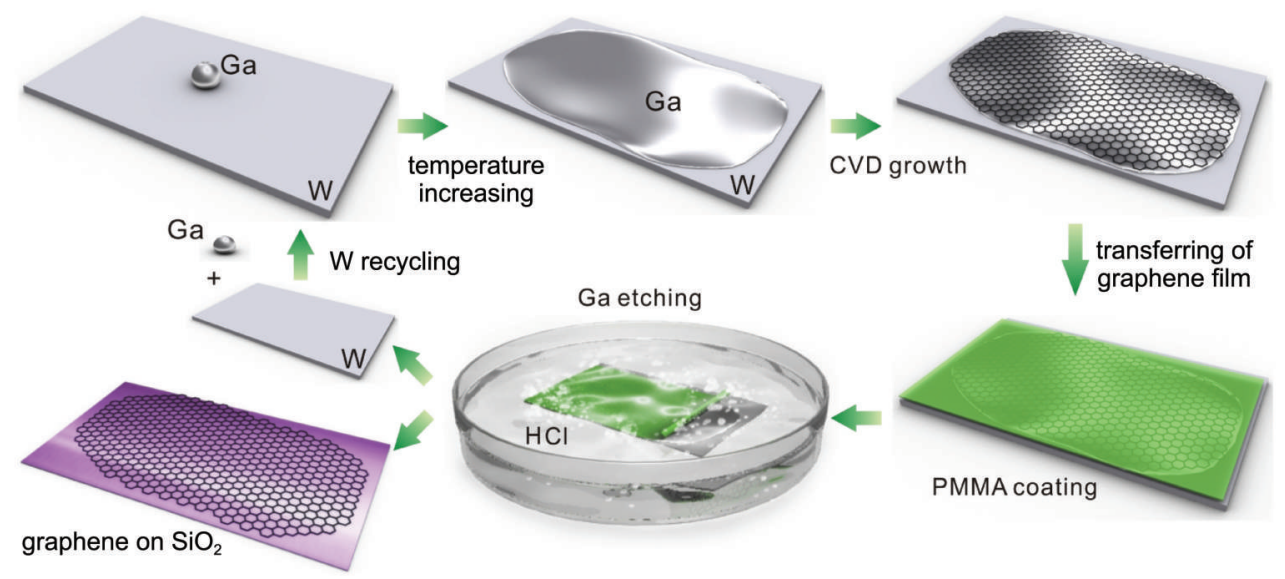

图 1 液态金属 $\mathbf{G a}$ 上石墨烯的生长与转移 ${ }^{15}$

Fig.1 Growth and transfer of graphene on liquid $\mathbf{G a}^{15}$

Schematic illustrates graphene growth and transfer over liquid Ga supported on a W substrate.

融态的金属(液态 $\mathrm{Cu}$ 、液态 In)表面石墨烯的生长行 为, 均获得了严格单层的石墨烯薄膜 ${ }^{29,30}$ 。研究表 明, 在碳源量、生长时间、生长温度以及降温速率 发生大幅改变时，在液态金属表面均可获得 100\% 覆盖的均匀单层石墨烯。这表明用液态金属生长 石墨烯时, 对生长参数变化具有较强的容错性。 我们对这一独特的自限制生长行为进行了分析, 并以 $\mathrm{Cu}$ 为例进行了说明。通过对生长完石墨烯后 的液态 $\mathrm{Cu}$ 以及固态 $\mathrm{Cu}$ 基底进行 $\mathrm{X}$ 射线光电子能谱
(XPS)深度分析, 发现这二者具有完全不同的溶碳 性。相比于几乎不溶碳的固态 $\mathrm{Cu}$, 液态 $\mathrm{Cu}$ 内部含 有大量的碳原子, 这归因于熔化后 $\mathrm{Cu}$ 原子之间较 大的空隙。而在降温过程中, 凝固从液态金属表 面开始发生, 表层先变为不溶碳的固态, 封住了 金属内部碳原子向金属表面析出的路径(图3)。这 使得石墨烯的生长严格遵循表面自限制机理, 同 时也使得生长条件变化对石墨烯的生长影响较小。

此外, 液态金属催化剂还可以用于辅助二维 (a)

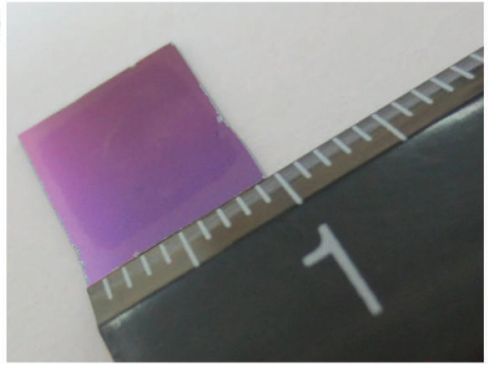

(b)

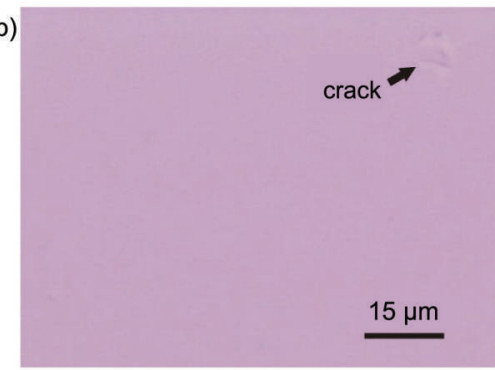

(d)

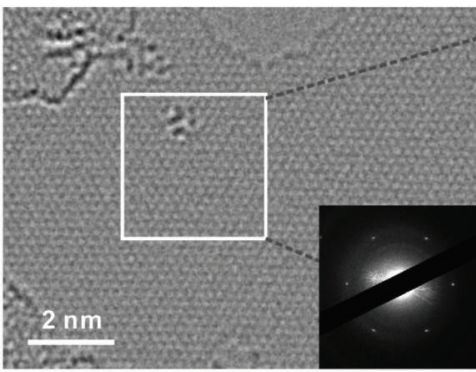

$-\ldots$

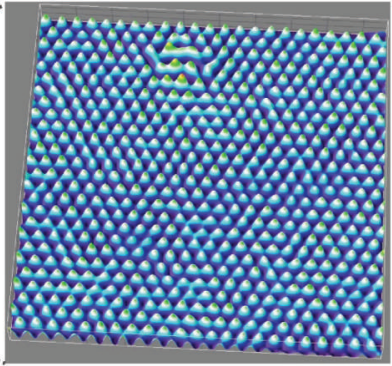

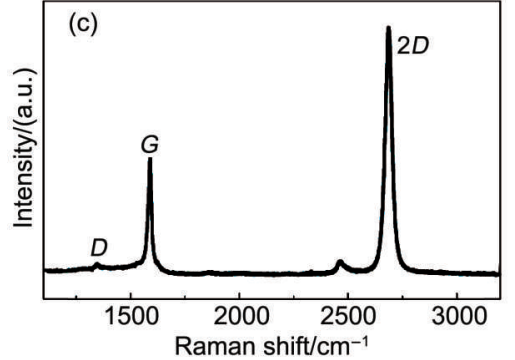

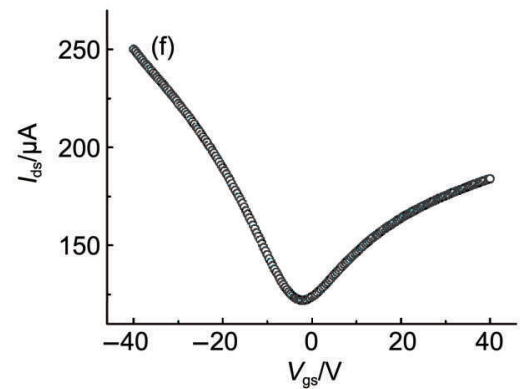

图 2 液态金属 $\mathbf{G a}$ 上生长的石墨烯的表征 ${ }^{15}$

Fig.2 Characterization of graphene grown on liquid metal $\mathrm{Ga}^{15}$

(a) graphene film transferred onto a $\mathrm{SiO}_{2} / \mathrm{Si}$ substrate; (b) optical image of the transferred graphene on a $\mathrm{SiO}_{2} / \mathrm{Si}$ substrate;

(c) Raman spectrum of the single-layer graphene; (d) HRTEM image and the selected-area electron diffraction pattern of the single-layer graphene and (e) a false 3D image originated from Fourier enhanced TEM micrograph; (f) the typical transfer characteristic of the fabricated FET based on the graphene grown on liquid $\mathrm{Ga}$, the $I_{\mathrm{ds}}$ means the drain-source current and the $V_{\mathrm{gs}}$ means the gate-source voltage. 


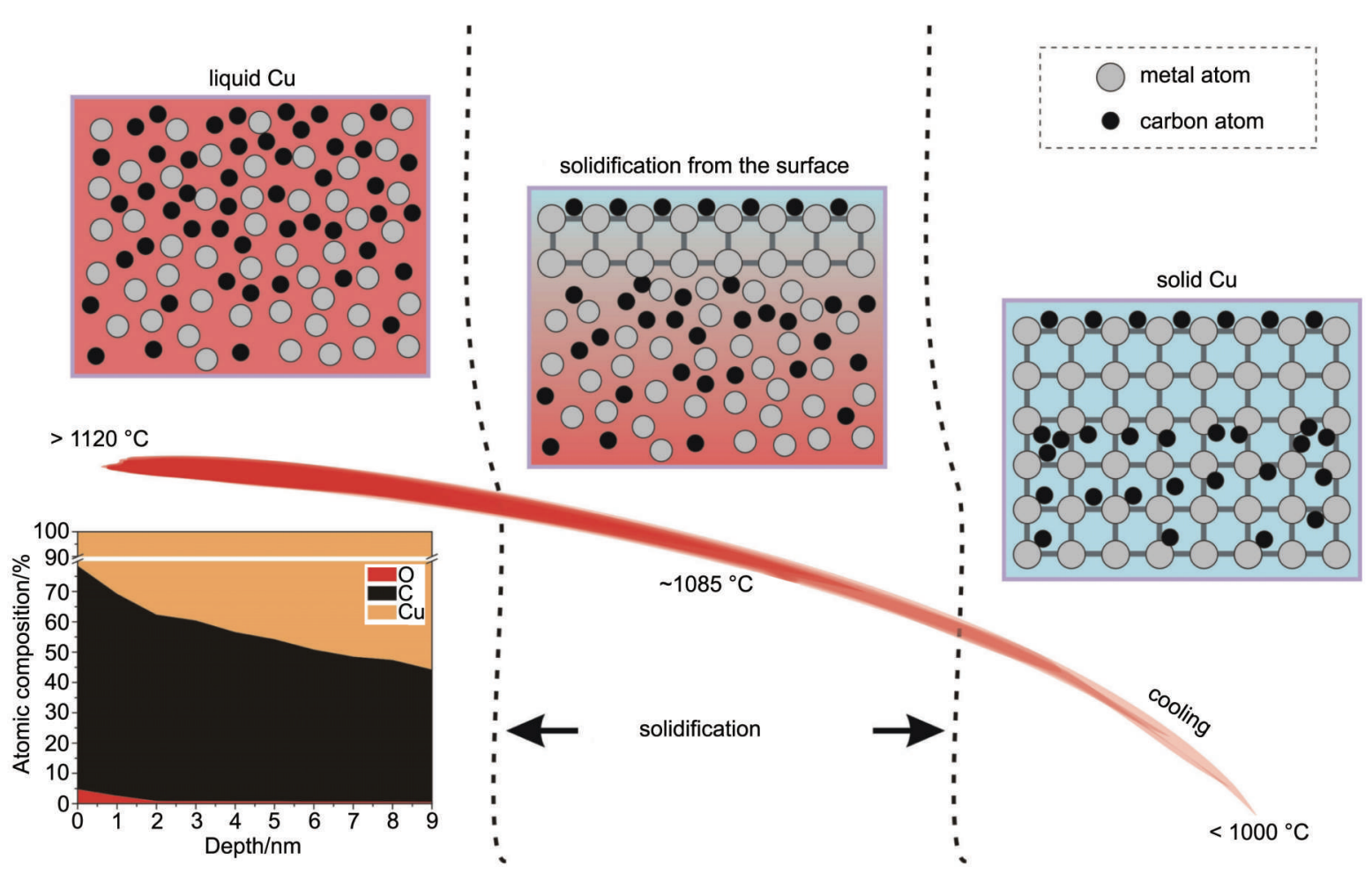

图 3 液态金属基底上生长严格单层石墨烯的机理 ${ }^{29}$

Fig.3 Mechanism for the growth of strictly monolayer graphene on liquid metal substrate ${ }^{29}$

Schematic illustrates the carbon distribution in liquid $\mathrm{Cu}$, solid $\mathrm{Cu}$, and the solidified surface of liquid $\mathrm{Cu}$ during the phase transition.

材料在绝缘基底上实现自限制生长。免转移直接 在目标基底上生长二维材料不仅可以避免转移过 程所带来的污染和破损, 还可以避免层间渗入杂 质分子, 从而增强二维材料与目标基底的相互作 用。然而, 通常使用的目标基底对二维材料的生 长(特别是石墨烯)并不具备良好的催化活性, 导致 所生长的二维材料质量不佳且层数控制困难。本 课题组创新性地利用液态金属蒸气辅助催化石墨 烯生长, 为实现绝缘基底上高质量石墨烯生长开 辟了新路径 ${ }^{31,32}$ 。这一方法不仅可以提高石墨烯的 质量、缩短生长时间、降低生长温度, 最为重要 的是, 实现了大面积均匀的石墨烯在绝缘基底上 的自限制生长。将 $\mathrm{Ga}$ 球置于生长石墨烯的石英基 底的上游, 利用 $\mathrm{Ga}$ 蒸气催化碳源分解, 显著提高 了石墨烯的生长效率, 在石英基底上成功获得了 大面积连续的单层石墨烯薄膜 (图 4) ${ }^{31}$ 。二维材料的 生长通常包含传质过程与反应过程, 已有的研究 表明: 未加催化剂时, 前驱体的传质速度快而反 应速度慢, 这就导致在基底表面会有多余的前驱 体分子积累, 使其容易吸附在已形成的材料表面 而继续生长为厚层。金属蒸气辅助催化方法成功 实现了绝缘基底上二维材料的大面积自限制生
长, 并提高了生长效率, 推动了二维材料在应用 之路上的行进步伐。

\section{2 液态金属上二维材料的低温生长}

由于液态催化剂具有较高传质速率, 因而较 之在固态催化剂表面, 二维材料在液态催化剂表 面的生长具有完全不同的决速步, 这使得液态催 化剂在催化二维材料生长时具有一些独特的行 为。我们发现, 将液态金属 $\mathrm{Ga}$ 加入到传统的 $\mathrm{Cu}$ (Ni) 催化剂中, 可获得高催化活性的液态 $\mathrm{Cu}-\mathrm{Ga}$ (或 Ni-Ga) 合金, 能在较低的温度下催化生长高质 量的石墨烯薄膜 ${ }^{33,34}$ 。

通常的催化剂均需要在 $1000{ }^{\circ} \mathrm{C}$ 以上才能催化 生长出高质量的石墨烯, 这无疑增加了工业生产 时的能耗和工艺复杂度。我们课题组将液态金属 $\mathrm{Ga}$ 与具有高催化活性的固态催化剂(如 $\mathrm{Cu} 、 \mathrm{Ni}$ 、 $\mathrm{Fe} 、 \mathrm{Co}$ 等)相结合, 制得了 $\mathrm{Ga}-\mathrm{M}(\mathrm{M}=\mathrm{Cu}, \mathrm{Ni}, \mathrm{Fe}$, $\mathrm{Co}$ )合金催化剂, 成功地在 $600{ }^{\circ} \mathrm{C}$ 获得了大面积均 匀的高质量单层石墨烯, 显著降低了石墨烯生长 所需的温度 ${ }^{33,34}$ 。研究表明, 这种新型的 Ga-M 合金 催化剂具有极高的催化活性, 能够在低温下催化 碳源的裂解以及石墨烯的形成(图 5(a, b))。此外, 这样的液态合金体系依旧延续了液态催化剂表面 


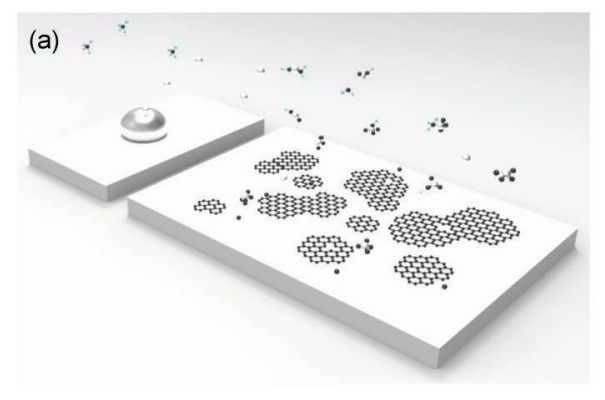

(b)
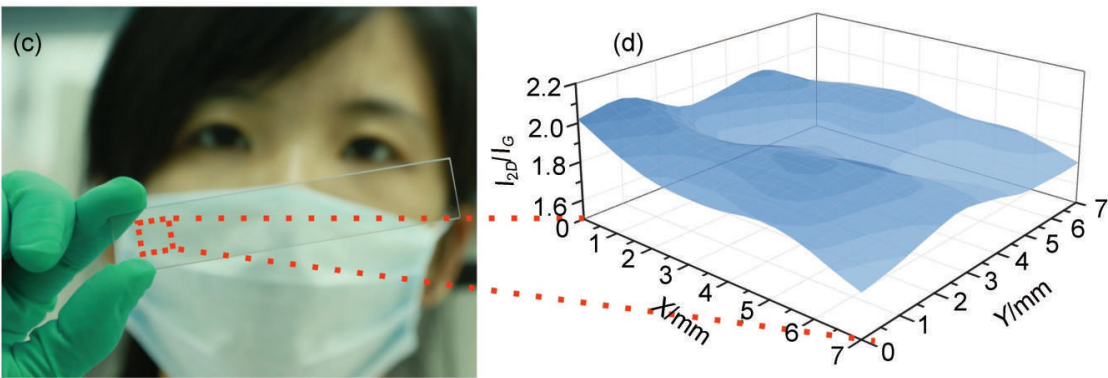

图 4 液态金属辅助在绝缘基底上自限制生长均匀层数石墨烯 ${ }^{31}$

Fig.4 Self-limited growth of uniform graphene on insulating substrate assisted by liquid metal ${ }^{31}$

(a) schematic drawing of Ga vapor-assisted CVD directly grown graphene on a quartz substrate;

(b) optical microscope image of the uniform graphene film transferred onto $\mathrm{SiO}_{2} / \mathrm{Si}$; (c) photograph of the grown graphene film on quartz substrate;

(d) Raman mapping result acquired from the dotted area in (c) revealed the uniformity of graphene
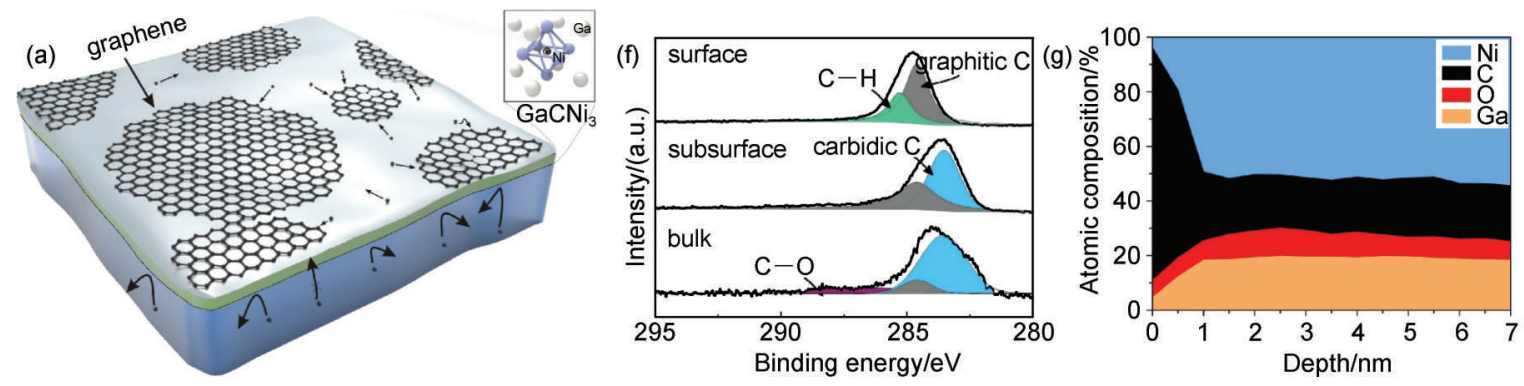

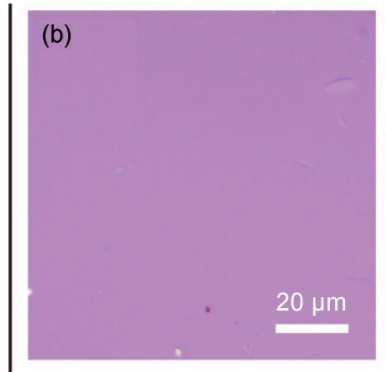

growth temperature $\left(600-1000^{\circ} \mathrm{C}\right)$

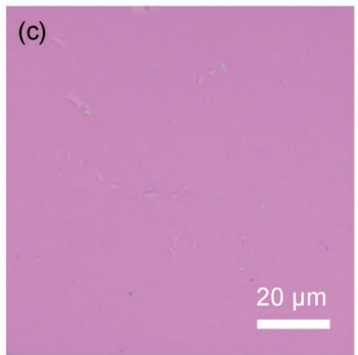

carbon source

$\left(\mathrm{CH}_{4}: 25-600 \mathrm{~mL}\right)$ (d)

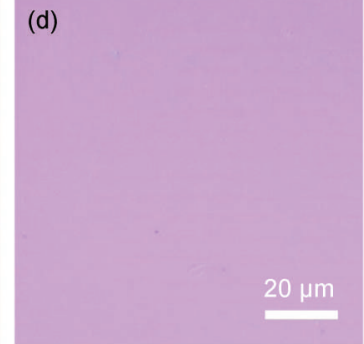

growth time

$(5-120 \mathrm{~min})$

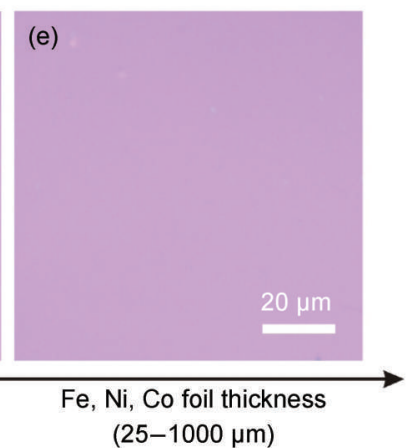

图 5 液态合金基底上石墨烯的低温生长 ${ }^{34}$

Fig.5 Growth of graphene on liquid alloy substrate at reduced temperature ${ }^{34}$

(a) schematic illustration of graphene growth over liquid alloy; (b-e) typical OM (optical microscopy) images of graphene films grown on galliumcovered iron (or cobalt or nickel) foils under different growth conditions; (f) X-ray photoelectron spectroscopy (XPS) analyses of C $1 s$ peaks from surface and bulk on Ga-Ni after the graphene growth; (e) XPS composition profiles of elements along the surface normal direction on Ga-Ni foil

利于生长均匀石墨烯的优势, 当生长温度、碳源 量、生长时间、金属 $\mathrm{M}$ 含量发生改变时, 在 $\mathrm{Ga}-\mathrm{M}$ 合金表面均可获得 100\%覆盖的均匀单层石墨烯(图 $5(b-e))$ 。对生长石墨烯后的合金基底进行 XPS 深
度分析发现: 合金中的 $\mathrm{Ga}$ 能够将体相中多余的碳 源以三元合金(如 $\mathrm{GaCNi}_{3}$ ) 的形式封存在内部(图 5 $(\mathrm{f}, \mathrm{g}))$, 从而有效降低表面碳源浓度, 实现了均匀 单层石墨烯在低温条件下的生长。 


\section{3 液态金属上二维材料异质结的直接构筑}

二维材料面间异质结因其具有独特、优异的 光、电性能, 近几年成为研究热点。目前构建面 间异质结大多需要经历多次机械剥离, 难以实现 异质结的大规模制备, 且这种方法构建的异质结 层间作用力较弱。直接生长出二维材料面间异质 结是其迈向实际实用的关键。近期，本课题组采 用抗硫化 $\mathrm{Ni}-\mathrm{Ga}$ 合金作为生长基底实现了 $\mathrm{MoS}_{2} / \mathrm{h}$ BN 异质结的直接生长, 无需任何转移步骤 35 。 Ni$\mathrm{Ga}$ 合金基底对 h-BN 表现出很好的催化活性，同时 其优良的抗硫化性能可以避免 $\mathrm{h}-\mathrm{BN}$ 生长时基底被 $\mathrm{H}_{2} \mathrm{~S}$ 破坏, 使 $\mathrm{MoS}_{2} / \mathrm{h}-\mathrm{BN}$ 面间异质结的直接构筑成 为可能, 如图 6(a) 所示。这种策略制备的 $\mathrm{MoS}_{2} / \mathrm{h}$ $\mathrm{BN}$ 异质结具有紧密的面间接触(图 6(b))。直接生 长于 h-BN 上的 $\mathrm{MoS}_{2}$ 面积可达 $200 \mu \mathrm{m}^{2}$ (图 6(c)), 比 前人的报道 $\left(\sim 10 \mu \mathrm{m}^{2}\right)$ 提高了一个数量级 ${ }^{36}$; 直接带 隙达到 $1.85 \mathrm{eV}$, 接近机械剥离的样品, 迁移率为 $22 \mathrm{~cm}^{2} \cdot \mathrm{V}^{-1} \cdot \mathrm{s}^{-1}$, 开关比达到 $10^{6}$ (图 6(d))。通过设 计液态金属合金的组份, 可以对催化活性和成核 生长行为进行调控, 构建一些传统方法难以获得 的新的异质结构, 这将极大拓展液态金属这一新 兴催化剂的应用范围，使其成为二维材料生长方 面的 “万能钥匙”。

此外, 本课题组还利用液态金属首次实现了 $100 \%$ 重叠的过渡金属二硫族化合物(TMDCs) 面间 异质结的可控制备 ${ }^{37}$ 。溶有少量过渡金属(如 Mo、
$\mathrm{W}$ 和 $\mathrm{Re}$ 等)的 $\mathrm{Au}$ 基底对 $\mathrm{TMDCs}$ 生长具有良好的催 化活性, 且 TMDCs 的生长表现出新颖的伴生生长 行为。以 $\mathrm{ReS}_{2}$ 和 $\mathrm{WS}_{2}$ 面间异质结的生长为例：采 用密度泛函理论计算表明, $\mathrm{ReS}_{2}$ 的生长只能在 $\mathrm{WS}_{2}$ 的(001)面进行, 从而使得它表现出与 $\mathrm{WS}_{2}$ 共生的 特殊生长行为, 最终实现了这种 $100 \%$ 重叠的 $\mathrm{ReS}_{2} /$ $\mathrm{WS}_{2}$ 面间异质结的可控生长, 如图 7 所示。通过这 种方法生长出的异质结不存在界面污染以及氧化物 残留的问题。此外，这种方法还有望实现其他多种 TMDCs 面间异质结的可控生长, 有望推动有关各 种二维异质结及超晶格结构的生长及理论研究。

\section{4 液态金属上二维材料的组装}

小至原子、单分子, 大到复杂的多级结构, 材料的自组装一直是一个非常热门的话题。自组 装成宏观体或者高级有序结构对于材料的应用十 分重要。以石墨烯为代表的二维原子晶体材料具 备优异的物理、化学性质, 若能实现其自组装将 促进其大规模应用进程。一般而言，在设计自组 装过程时需要关注两个方面: 结构单元间的相互 作用(如氢键、范德华力、静电力等)和外加的驱动 力 $(\text { 静电场、磁场、流变场等 })^{38}$ 。目前尚无任何关 于高质量二维单晶的自组装的报道, 这无疑是个 缺憾。究其原因, 是因为目前 CVD 法制得的二维 材料单晶均是附着于固态基底的，晶体一旦形 核, 便只能原位长大, 外加的驱动力无法驱动一

(a)
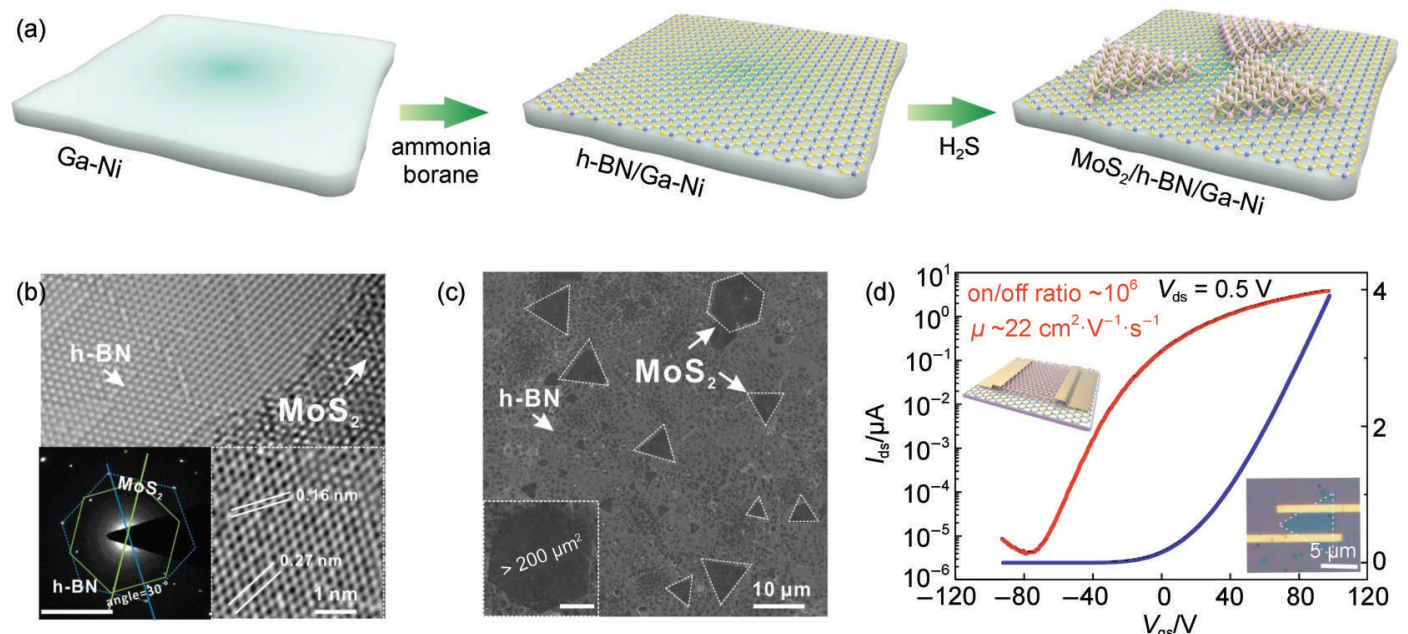

(c)

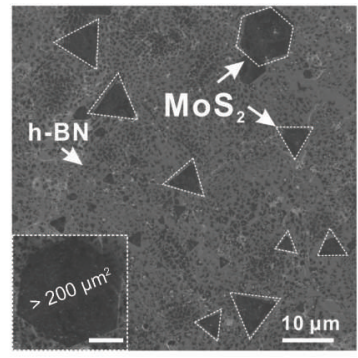

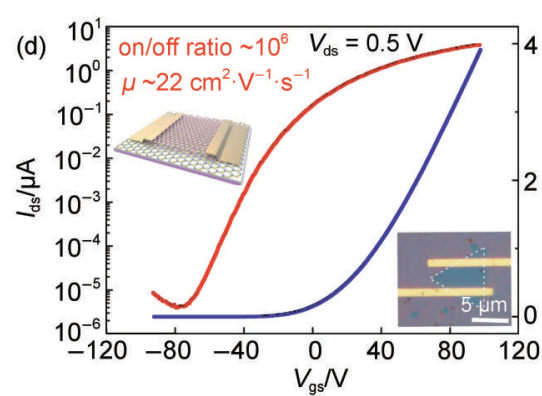

图 6 液态抗硫化合金基底上 $\mathrm{MoS}_{2} / \mathrm{h}-\mathrm{BN}$ 异质结的生长及表征 ${ }^{35}$

Fig.6 Growth of $\mathrm{MoS}_{2} / \mathrm{h}-\mathrm{BN}$ heterostructure on liquid sulfide-resistant alloy substrate and its characterizations $\mathrm{s}^{35}$

(a) schematic showing the preparation of TMDCs/h-BN (transition metal dichalcogenides/hexagonal boron nitride) heterostructures;

(b) TEM characterizations of $\mathrm{MoS}_{2} / \mathrm{h}-\mathrm{BN}$ heterostructures. Inset shows SAED patterns corresponding to the heterostructures;

(c) SEM image of the directly grown single-crystal $\mathrm{MoS}_{2}$ on h-BN. Inset shows $\mathrm{MoS}_{2}$ crystal with grain size up to $200 \mu \mathrm{m}^{2}$;

(d) electrical properties of $I_{\mathrm{ds}}-V_{\mathrm{gs}}$ curves for the FET devices made on $\mathrm{MoS}_{2} / \mathrm{h}-\mathrm{BN}$ heterostructures. Inset shows optical image of the device. 


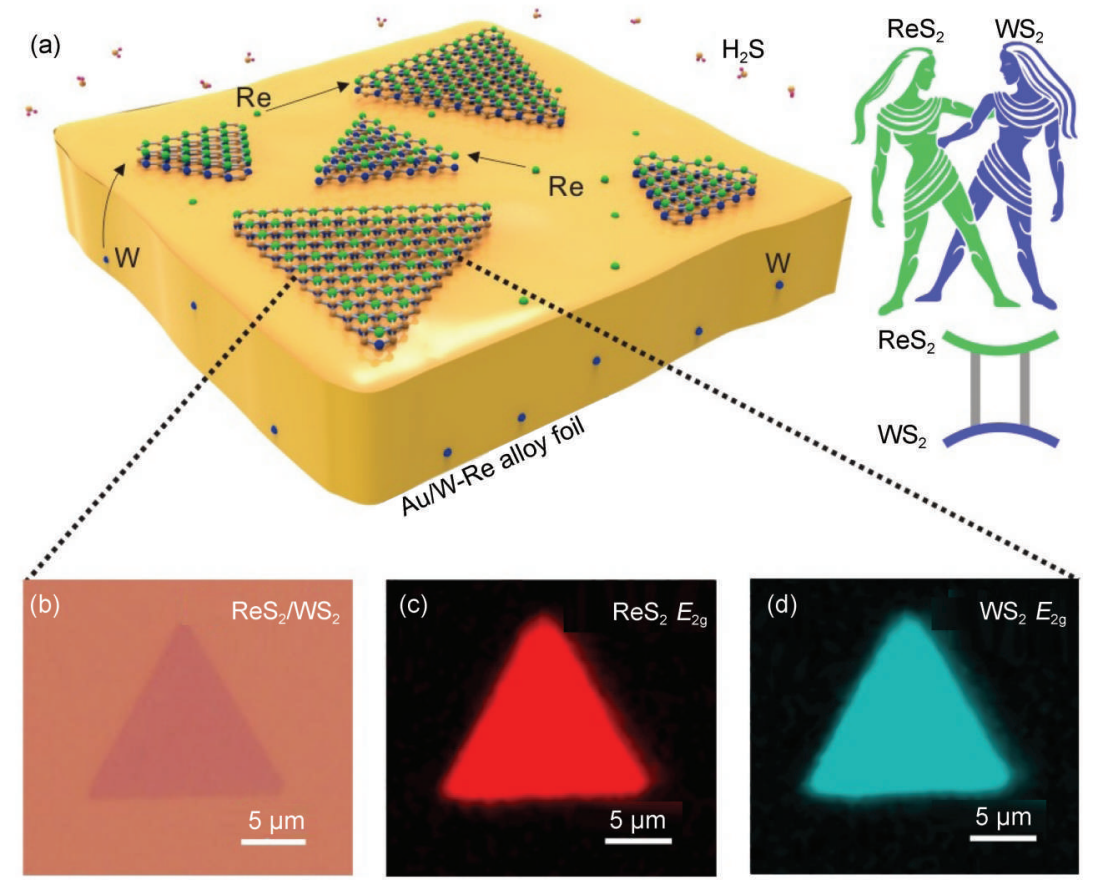

图 7 液态 $\mathrm{Au}$ 法共生生长 $100 \%$ 重叠的 $\mathrm{ReS}_{2} / \mathrm{WS}_{2}$ 面间异质结及其表征 ${ }^{37}$

Fig.7 Twinned growth of $100 \%$ overlapped $\mathrm{ReS}_{2} / \mathrm{WS}_{2}$ vertical heterostructures based on liquid Au strategy and their characterizations ${ }^{37}$

(a) schematic for the twinned growth of vertically stacked $\mathrm{ReS}_{2} / \mathrm{WS}_{2}$ heterostructures;

(b) typical OM of a $\mathrm{ReS}_{2} / \mathrm{WS}_{2}$ twinned vertical heterostructure crystal; (c, d) Raman mappings of peak intensity at 160.5 and $351.3 \mathrm{~cm}^{-1}$, respectively, corresponding to the $E_{2 \mathrm{~g}}$ mode of $\mathrm{ReS}_{2}$ and $\mathrm{WS}_{2}$, respectively.

个非流变相中的单元发生自组装, 单晶间的相互 作用力也未被认识。本课题组长期研究的液态金 属催化剂有望为高质量二维单晶的组装带来福音。

\section{1 平滑拼接}

CVD 法制得的石墨烯薄膜实际上是由众多石 墨烯晶畴无规拼接组装而成, 内部含有大量晶 界。如果能实现晶畴间平滑拼接(无缝组装), 无疑 对于提高大面积石墨烯膜的整体质量至关重要。 本课题组利用液态基底表面各向同性的特点, 研 究了石墨烯的生长、拼接行为, 提出了一种新的 思路来实现石墨烯单晶的平滑拼接组装 ${ }^{39}$ 。液态基 底对石墨烯生长无诱导取向, 且其表面良好的流 动性有助于实现石墨烯的各向同性生长, 由此可 得到通常方法难以获得的圆形石墨烯单晶。这种 特殊形状的单晶边缘呈细密的锯齿状, 原子排列 为混合的 zigzag 型和 armchair 型。相较于六边形的 单晶, 圆形单晶在发生旋转时位阻较小, 而且边 缘表现出非常高的反应活性。在液态金属这样一 个可流动的平台上, 圆形石墨烯晶粒仅需转动一 个很小的角度, 即可实现与相邻晶粒间的平滑拼 接, 以达到能量最小化(如图 8 所示)。由此, 晶界
导致的负面效应可以被大幅地削弱, 这一点通过 测试跨晶界处和单晶内的迁移率获得了证实。

\section{2 有序组装}

若能将高质量二维材料单晶自组装为超有序 的周期性结构, 无疑对于其在光、电器件中的后 续应用极具吸引力。在液态金属这样一个可流变 的平台上, 我们利用气流扰动和自主播种策略, 以石墨烯为例, 实现了高质量单晶的自组装, 首 次获得了二维材料的超有序结构(SOS)(如图 9(a,b) 所示 $)^{40}$ 。而且有序结构中的石墨烯单晶的取向也是 高度一致的(如图 9(c)所示), 这得益于石墨烯生长 过程中产生的各向异性且大小均一的静电相互作 用力。此外, 通过改变扰动气流的大小, 石墨烯 超有序结构的周期性可以被精确调控; 通过固态 碳源的设计, 石墨烯单元的化学性质可以被有效 调节, 从而满足未来不同的应用需求。

我们还发现这样的自组装行为还可拓展至其 它二维材料, 如六方氮化硼 $(\mathrm{h}-\mathrm{BN})^{41}$ 。与石墨烯类 似, 液态 $\mathrm{Cu}$ 表面生长的 $\mathrm{h}-\mathrm{BN}$ 单晶也呈现出各向同 性的圆形, 其边缘为交替排列的 $\mathrm{B}$ 和 $\mathrm{N}$ 原子, 而不 是通常大家所认为的能量最低的 $\mathrm{N}$ 端终止形态(表 
(a)

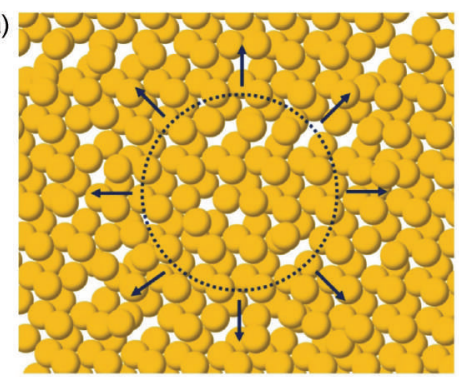

(d)

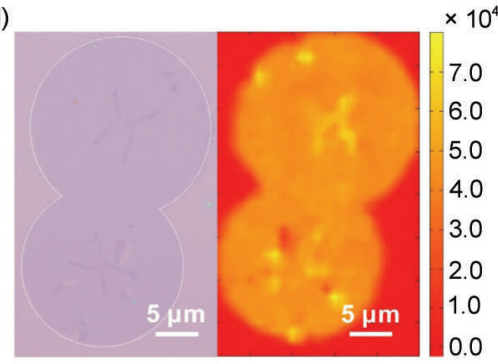

(b)
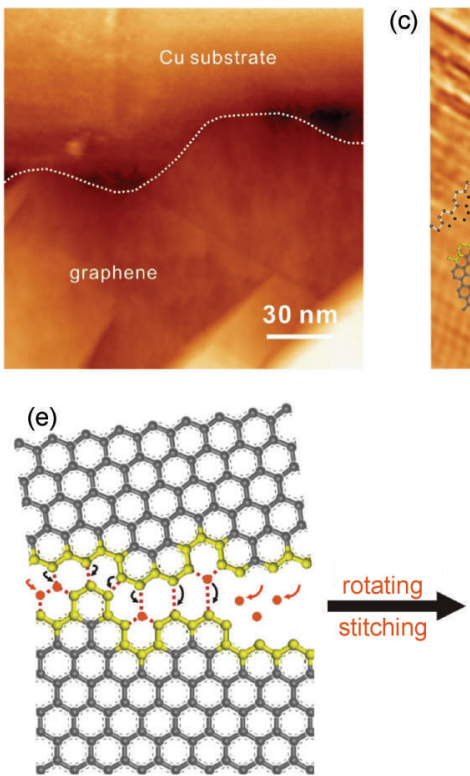

(c)
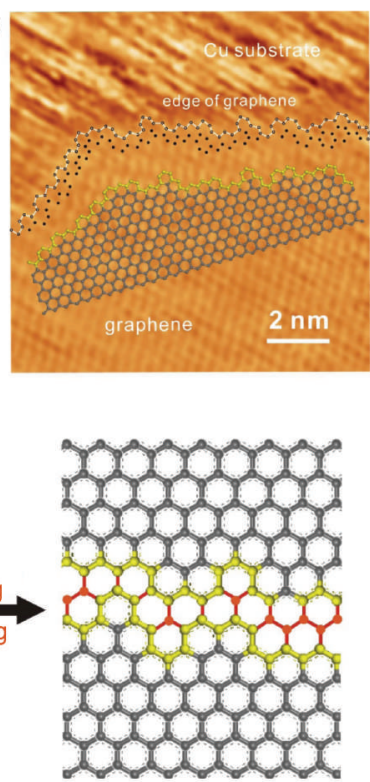

图 8 液态 $\mathrm{Cu}$ 上石墨烯的各向同性生长及晶粒间的平滑拼接组装 ${ }^{39}$

Fig.8 Isotropic growth of graphene and the smooth stitching of the grains on liquid $\mathrm{Cu}^{39}$

(a) scheme of the IGG (isotropic graphene grains) via isotropic growth on the liquid Cu; (b) typical STM image of the edge of an individual IGG;

(c) atom images at the edge of the IGG and the simulative atom structures derived from the actual atom arrangements;

(d) Raman mapping of $I_{2 D}$; (e) stitching process of two adjacent IGGs
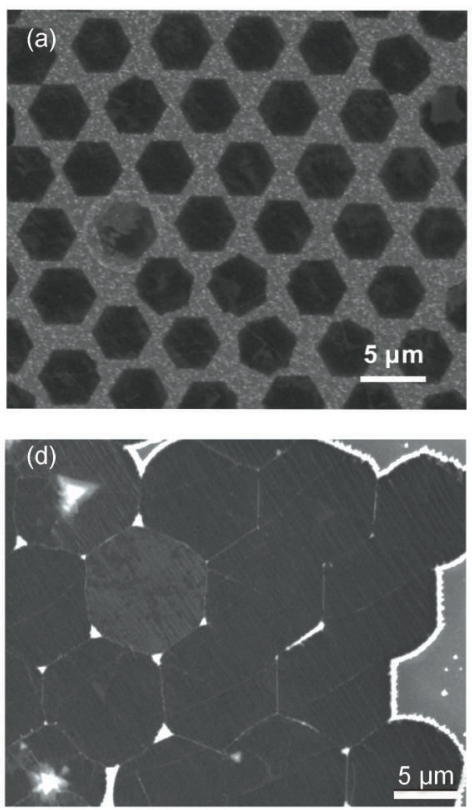
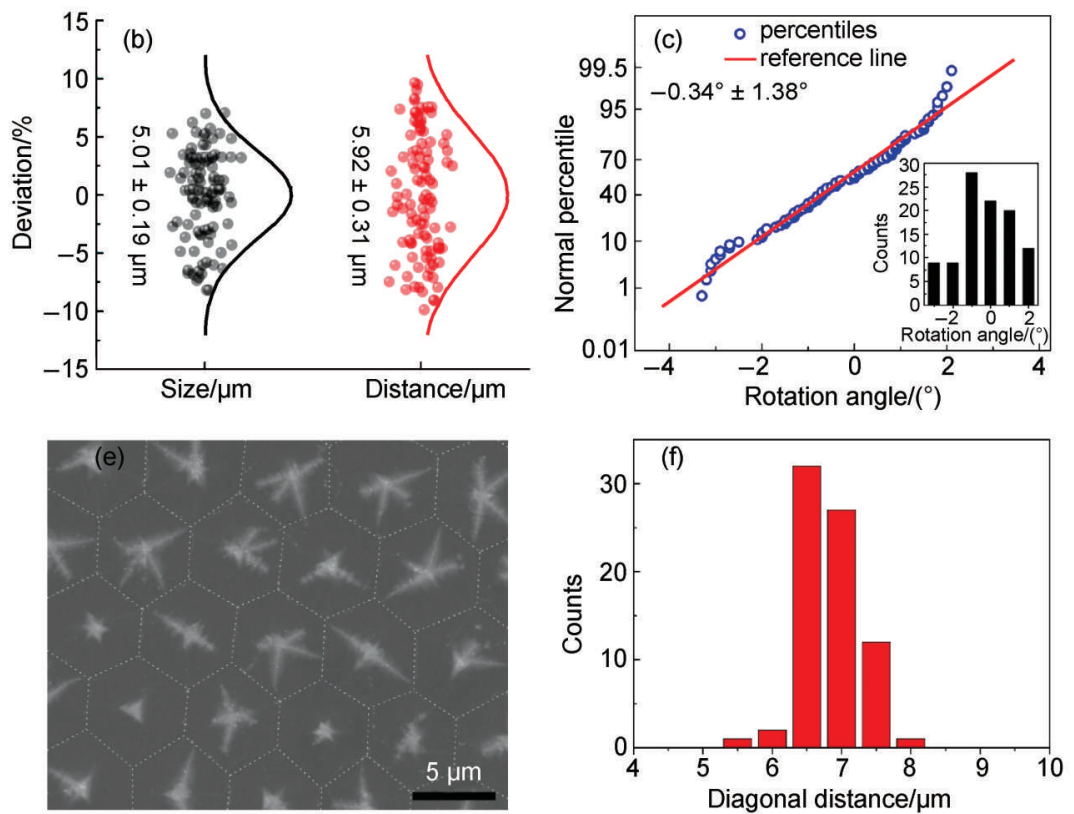

图9 液态金属表面二维材料单晶的超有序自组装 40,41

Fig.9 Super-ordered self-assembly of two-dimensional single crystals on liquid metal surface ${ }^{40,41}$

(a) SEM image of the graphene super-ordered structure (GSOS); (b) statistical distribution about the deviation of size and distance of GSOS; (c) percentile curve and (inset) histograms of the rotation angle of GSOS; (d) SEM image of the h-BN self-aligned single crystal array (SASCA); (e) SEM image of the h-BN SASCA with snowflakes visualized by the surplus growth; (f) typical diagram of the size distribution of h-BN single-crystals

现为三角形晶型)。基于这种高边缘活性的圆形 $\mathrm{h}-$ $\mathrm{BN}$ 单晶, 我们首次得到了自对准的单晶 h-BN阵 列(SASCA), 如图 9(d) 所示。通过加大前驱体的 量, 可以发生选区吸附, 从而实现单晶分布的可
视化(如图 9(e))。该阵列具有非常规整有序的排 布, 且单晶大小分布窄(如图 9(f))。我们还对该单 晶阵列进行了绝缘性能测试, 当施加电压从 -4 至 $4 \mathrm{~V}$ 时, 未检测到有明显电流通过, 表现出良好的 
(a)

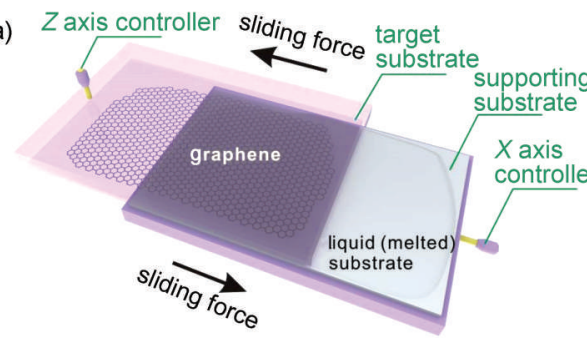

(c)

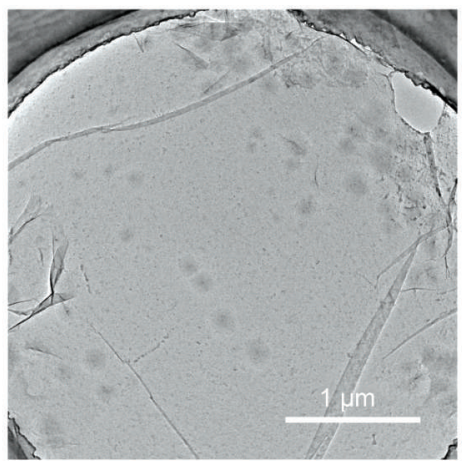

(e)

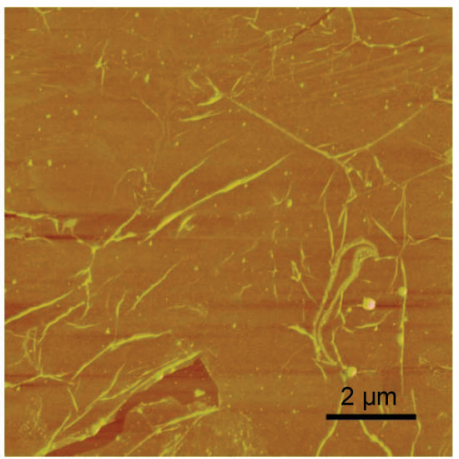

(b)

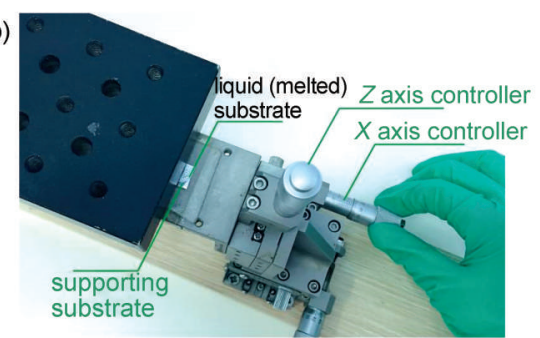

(d)

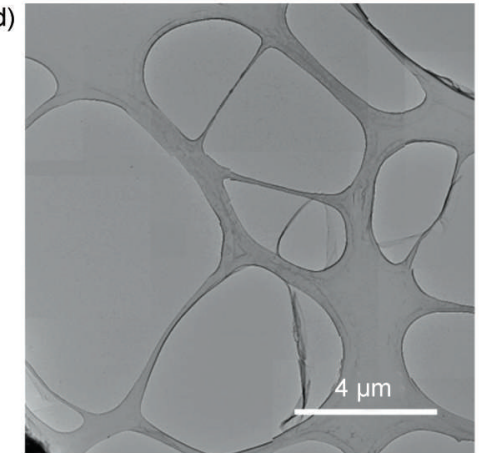

(f)

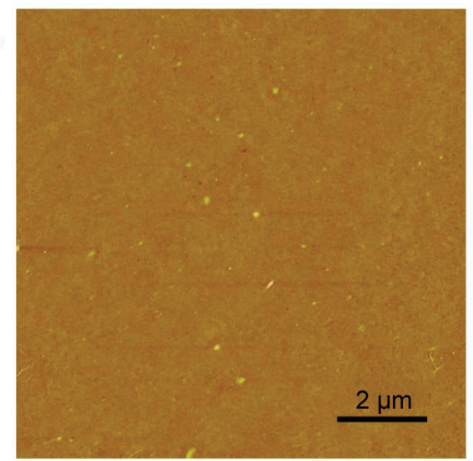

图 10 液态金属上石墨烯的直接滑移转移 ${ }^{42}$

\section{Fig.10 Direct sliding transfer of graphene grown on liquid metal ${ }^{42}$}

(a) The scheme shows the sliding process; (b) a photograph of the equipment for sliding transfer;

(c) TEM image of graphene transferred by traditional PMMA-assisted method; (d) TEM image of sliding transferred graphene;

(e) AFM image of PMMA-assisted transferred graphene on $\mathrm{SiO}_{2} / \mathrm{Si}$; (f) AFM image of sliding transferred graphene on $\mathrm{SiO}_{2} / \mathrm{Si}$

绝缘性, 有望应用于集成的石墨烯及其它二维材 料的场效应晶体管(FETs)中。

\section{5 液态金属上二维材料的转移}

相较于传统的固态金属, 液态金属催化剂在 二维材料的生长和组装方面表现出非常明显的优 势。而在后续的器件构筑过程中, 液态金属特殊 的物态也为二维材料的转移提供了新的思路。我 们摒弃传统方法必须引入聚合物载体辅助的固有 观念, 将液态金属上生长的石墨烯与转移目标基 底面对面地直接接触, 通过滑移将石墨烯直接转 移到了任意目标基底上, 如图 10 所示 ${ }^{42}$ 。两个关键 因素促成了这样的一个高保真的转移过程: 首 先, 金属呈熔融状态时其原子具有可易位性, 这 使得滑移转移过程中金属原子之间可以发生分
离; 其次, 温和的水平滑移力, 滑移时产生的侧 推力有助于石墨烯薄膜与目标基底之间更好的紧 贴。这种新型的滑移转移方法具有超快速(几秒)、 可控性高(均匀的层数, 大面积的和精确定位)和无 损(无褶皱、无裂纹、无污染)的特色，而且具有良 好的可拓展性, 同样适用于转移 h-BN 等其它二维 材料, 并且对目标基底的选择不挑剔, 这无疑将 推进石墨烯的基础研究和其未来的实际应用。

\section{6 总结与展望}

液态金属催化剂具有表面各向同性、体相原 子空位多、易于切变、流动性好等特点, 这使得 其用作二维材料 $\mathrm{CVD}$ 生长的基底时, 传质、催化 和组装行为均较之固态金属催化剂有很大区别。 目前液态金属催化剂已经在催化大面积、严格单 
层、高质量石墨烯生长时展现出巨大的优势。再 者, 这样的一个液态体系还具有极强的可拓展 性, 通过对液态组份进行设计, 可实现二维材料 低温生长和新型异质结的构筑。更为重要的是, 液态金属的流变性使得这样的一个平台能实现二 维材料生长和组装的一体化, 实现晶粒间的平滑 拼接和超有序排列, 带来了一种全新、无需任何 辅助载体的超洁净转移方法, 这是传统的固态金 属催化剂无可比拟的。我们相信液态金属催化剂 能带给我们的远不止已发现的这些。目前大多数 的液态金属 CVD 初探是以石墨烯为例的, 其它更 多的二维材料在液态基底上会带给我们哪些独特 生长与组装行为尚未可知, 对此我们充满期待。 尽管如此, 液态金属催化剂在催化二维材料生长 时也面临着一些问题。传统的可用于催化二维材 料生长的过渡金属催化剂的原子的最外层 $s$ 电子和 $d$ 电子均可参与成键, 金属键的强度很高, 一般都 具有很高的熔点, 比如 $\mathrm{Cu}$ 的熔点为 $1083{ }^{\circ} \mathrm{C}, \mathrm{Ni}$ 的熔点更是高达 $1453{ }^{\circ} \mathrm{C}$, 这无疑对将其液态化是 一个很大的阻碍, 不利于它们未来的工业化应 用。此外, 由于液态表面无定形的特点, 其表面 无特定的晶格结构, 这样一个复杂的体系使得关 于其催化机制的理论研究仍十分贵乏。我们难以 将研究固态催化剂的一些方法, 比如密度泛函理 论计算, 直接用于研究液态表面结构对 CVD 基元 步骤(如碳原子的吸脱附)的影响 ${ }^{43}$ 。再者, 较之固 态金属催化剂, 更为丰富的反应或传质界面, 如 气-固界面、液-固界面, 气-液界面等, 也使得液 态金属催化体系的催化机制更为复杂。而目前关 于液态金属催化剂的研究大多还停留在实验验证 阶段, 如能建立更为系统的理论指导和验证体 系, 无疑将更进一步促进液态金属 CVD 对二维材 料可控制备的发展。我们可以预期液态金属催化 剂将成为推动二维材料研究与应用的一股巨大力 量。

\section{References}

(1) Miro, P.; Audiffred, M.; Heine, T. Chem. Soc. Rev. 2014, 43, 6537. doi: 10.1039/c4cs00102h

(2) Katsnelson, M. I. Mater. Today 2007, 10, 20. doi: 10.1016/ S1369-7021(06)71788-6

(3) Zhang, Y.; Tang, T. T.; Girit, C.; Hao, Z.; Martin, M. C.; Zettl, A.; Crommie, M. F.; Shen, Y. R.; Wang, F. Nature 2009, 459, 820. doi: $10.1038 /$ nature 08105
(4) Ohta, T.; Bostwick, A.; Seyller, T.; Horn, K.; Rotenberg, E. Science 2006, 313, 951. doi: 0.1126/science. 1130681

(5) Geim, A. K.; Novoselov, K. S. Nat. Mater. 2007, 6, 183. doi: 10.1038/nmat 1849

(6) Craciun, M.; Russo, S.; Yamamoto, M.; Oostinga, J. B.; Morpurgo, A.; Tarucha, S. Nat. Nanotechnol. 2009, 4, 383. doi: 10.1038/nnano.2009.89

(7) Yu, Q. K.; Jauregui, L. A.; Wu, W.; Colby, R.; Tian, J. F.; Su, Z. H.; Cao, H. L.; Liu, Z. H.; Pandey, D.; Wei, D. G.; Chung, T. F.; Peng, P.; Guisinger, N. P.; Stach, E. A.; Bao, J. M.; Pei, S. S.; Chen, Y. P. Nat. Mater. 2011, 10, 443. doi: 10.1038/nmat3010

(8) Ferrari, A. C.; Bonaccorso, F.; Fal'ko, V.; et al. Nanoscale 2015, 7, 4598. doi: 10.1039/c4nr01600a

(9) Reina, A.; Jia, X.; Ho, J.; Nezich, D.; Son, H.; Bulovic, V.; Dresselhaus, M. S.; Kong, J. Nano Lett. 2009, 9, 30. doi: $10.1021 / \mathrm{nl} 801827 \mathrm{v}$

(10) Liu, N.; Fu, L.; Dai, B. Y.; Yan, K.; Liu, X.; Zhao, R. Q.; Zhang, Y. F.; Liu, Z. F. Nano Lett. 2011, 11, 297. doi: 10.1021/ nl103962a

(11) Xue, Y. Z.; Wu, B.; Guo, Y. L.; Huang, L. P.; Jiang, L.; Chen, J. Y.; Geng, D. C.; Liu, Y. Q.; Hu, W. P.; Yu, G. Nano Res. 2011, 4, 1208. doi: 10.1007/s12274-011-0171-4

(12) Gall, N. R.; Rut'kov, E. V.; Tontegode, A. Y. Phys. Solid State 2004, 46, 371. doi: 10.1134/1.1649439

(13) Li, X. S.; Cai, W. W.; An, J. H.; Kim, S.; Nah, J.; Yang, D. X.; Piner, R.; Velamakanni, A.; Jung, I.; Tutuc, E.; Banerjee, S. K.; Colombo, L.; Ruoff, R. S. Science 2009, 324, 1312. doi: 10.1126/science.1171245

(14) Li, X. S.; Cai, W. W.; Colombo, L.; Ruoff, R. S. Nano Lett. 2009, 9, 4268. doi: 10.1021/n1902515k

(15) Wang, J.; Zeng, M.; Tan, L.; Dai, B.; Deng, Y.; Rümmeli, M.; Xu, H.; Li, Z.; Wang, S.; Peng, L.; Eckert, J.; Fu, L. Sci. Rep. 2013, 3, 2670. doi: 10.1038/srep02670

(16) Liu, X.; Fu, L.; Liu, N.; Gao, T.; Zhang, Y.; Liao, L.; Liu, Z. J. Phys. Chem. C 2011, 115, 11976. doi: 10.1021/jp202933u

(17) Wu, Y.; Chou, H.; Ji, H.; Wu, Q.; Chen, S.; Jiang, W.; Hao, Y.; Kang, J.; Ren, Y.; Piner, R. D.; Ruoff, R. S. ACS Nano 2012, 6 , 7731. doi: $10.1021 / \mathrm{nn} 301689 \mathrm{~m}$

(18) Weatherup, R. S.; Bayer, B. C.; Blume, R.; Ducati, C.; Baehtz, C.; Schlögl, R.; Hofmann, S. Nano Lett. 2011, 11, 4154. doi: $10.1021 / \mathrm{nl} 202036 \mathrm{y}$

(19) Dai, B.; Fu, L.; Zou, Z.; Wang, M.; Xu, H.; Wang, S.; Liu, Z. Nat. Commun. 2011, 2, 522. doi: 10.1038/ncomms 1539

(20) Zou, Z. Y.; Fu, L.; Song, X. J.; Zhang, Y. F.; Liu, Z. F. Nano Lett. 2014, 14, 3832. doi: 10.1021/n1500994m

(21) Geng, D. C.; Wu, B.; Guo, Y. L.; Huang, L. P.; Xue, Y. Z.; Chen, J. Y.; Yu, G.; Jiang, L.; Hu, W. P.; Liu, Y. Q. Proc. Natl. Acad. Sci. U. S. A. 2012, 109, 7992. doi: 10.1073/pnas.1200339109

(22) Geng, D. C.; Wang, H. P.; Yu, G. Adv. Mater. 2015, 27, 2821. doi: 10.1002/adma.201405887

(23) Luo, Z. T.; Lu, Y.; Singer, D. W.; Berck, M. E.; Somers, L. A.; 
Goldsmith, B. R.; Johnson, A. T. C. Chem. Mater. 2011, 23, 1441. doi: $10.1021 / \mathrm{cm} 1028854$

(24) Gan, L.; Luo, Z. T. ACS Nano 2013, 7, 9480. doi: 10.1021/ nn404393b

(25) Jung, D. H.; Kang, C.; Kim, M.; Cheong, H.; Lee, H.; Lee, J. S. J. Phys. Chem. C 2014, 118, 3574. doi: 10.1021/jp410961m

(26) Wang, H.; Xu, X.; Li, J.; Lin, L.; Sun, L.; Sun, X.; Zhao, S.; Tan, C.; Chen, C.; Dang, W.; Ren, H.; Zhang, J.; Deng, B.; Koh, A. L.; Liao, L.; Kang, N.; Chen, Y.; Xu, H.; Ding, F.; Liu, K.; Peng, H.; Liu, Z. Adv. Mater. 2016, 28, 8968. doi: 10.1002/ adma.201603579

(27) Zhang, Y.; Gomez, L.; Ishikawa, F. N.; Madaria, A.; Ryu, K.; Wang, C.; Badmaev, A.; Zhou, C. J. Phys. Chem. Lett. 2010, 1, 3101. doi: $10.1021 / \mathrm{jz} 1011466$

(28) Ogawa, Y.; Hu, B. S.; Orofeo, C. M.; Tsuji, M.; Ikeda, K.; Mizuno, S.; Hibino, H.; Ago, H. J. Phys. Chem. Lett. 2012, 3 , 219. doi: $10.1021 / \mathrm{jz} 2015555$

(29) Zeng, M.; Tan, L.; Wang, J.; Chen, L.; Rümmeli, M. H.; Fu, L. Chem. Mater. 2014, 26, 3637. doi: 10.1021/cm501571h

(30) Tan, L.; Zeng, M.; Zhang, T.; Fu, L. Nanoscale 2015, 7, 9105. doi: $10.1039 / \mathrm{c} 5 \mathrm{nr} 01420 \mathrm{~d}$

(31) Tan, L.; Zeng, M.; Wu, Q.; Chen, L.; Wang, J.; Zhang, T.; Eckert, J.; Rümmeli, M. H.; Fu, L. Small 2015, 11, 1840. doi: $10.1002 /$ smll.201402427

(32) Liu, J.; Zeng, M.; Wang, L.; Chen, Y.; Xing, Z.; Zhang, T.; Liu, Z.; Zuo, J.; Nan, F.; Mendes, R. G.; Chen, S.; Ren, F.; Wang, Q.; Rümmeli, M. H.; Fu, L. Small 2016, 12, 5741. doi: 10.1002/ smll.201601556

(33) Wang, J.; Chen, L.; Wu, N.; Kong, Z.; Zeng, M.; Zhang, T.; Zhuang, L.; Fu, L. Carbon 2016, 96, 799. doi: 10.1016/j. carbon.2015.10.015

(34) Chen, L.; Kong, Z.; Yue, S.; Liu, J.; Deng, J.; Xiao, Y.; Mendes,
R. G.; Rümmeli, M. H.; Peng, L.; Fu, L. Chem. Mater. 2015, 27, 8230. doi: 10.1021/acs.chemmater.5b02788

(35) Fu, L.; Sun, Y.; Wu, N.; Mendes, R. G.; Chen, L.; Xu, Z.; Zhang, T.; Rümmeli, M. H.; Rellinghaus, B.; Pohl, D.; Zhuang, L.; Fu, L. ACS Nano 2016, 10, 2063. doi: 10.1021/ acsnano. 5 b06254

(36) Wang, S. S.; Wang, X. C.; Warner, J. H. ACS Nano 2015, 9 , 5246. doi: 10.1021/acsnano.5b00655

(37) Zhang, T.; Jiang, B.; Xu, Z.; Mendes, R. G.; Xiao, Y.; Chen, L. F.; Fang, L. W.; Gemming, T.; Chen, S. H.; Rümmeli, M. H.; Fu, L. Nat. Commun. 2016, 7, 13911. doi: 10.1038/ncomms13911

(38) Parviz, B. A.; Ryan, D.; Whitesides, G. M. IEEE T. Adv. Packaging 2003, 26, 233. doi: 10.1109/tadvp.2003.817971

(39) Zeng, M.; Tan, L.; Wang, L.; Mendes, R. G.; Qin, Z.; Huang, Y.; Zhang, T.; Fang, L.; Zhang, Y.; Yue, S.; Rümmeli, M. H.; Peng, L.; Liu, Z.; Chen, S.; Fu, L. ACS Nano 2016, 10, 7189. doi: 10.1021/acsnano.6b03668

(40) Zeng, M.; Wang, L.; Liu, J.; Zhang, T.; Xue, H.; Xiao, Y.; Qin, Z.; Fu, L. J. Am. Chem. Soc. 2016, 138, 7812. doi: 10.1021/ jacs.6b03208

(41) Tan, L.; Han, J.; Mendes, R. G.; Rümmeli, M. H.; Liu, J.; Wu, Q.; Leng, X.; Zhang, T.; Zeng, M.; Fu, L. Adv. Electron. Mater. 2015, 1, 1500223. doi: 10.1002/aelm.201500223

(42) Lu, W.; Zeng, M.; Li, X.; Wang, J.; Tan, L.; Shao, M.; Han, J.; Wang, S.; Yue, S.; Zhang, T.; Hu, X.; Mendes, R. G.; Rümmeli, M. H.; Peng, L.; Liu, Z.; Fu, L. Adv. Sci. 2016, 3, 1600006. doi: 10.1002/advs.201600006

(43) Xu, X.; Zhang, Z.; Qiu, L.; Zhuang, J.; Zhang, L.; Wang, H.; Liao, C.; Song, H.; Qiao, R.; Gao, P.; Hu, Z.; Liao, L.; Liao, Z.; Yu, D.; Wang, E.; Ding, F.; Peng, H.; Liu, K. Nat. Nanotechnol. 2016, 11, 930. doi: 10.1038/NNANO.2016.132 